\title{
Sincronización cíclica en los países de la ALADI*
}

\section{CRISTIAN RABANAL}

CONICET- Universidad Nacional de Río Cuarto, Facultad de Ciencias Económicas, Of. 32, Ruta Nacional 36 Km. 601, 5800, Río Cuarto, Argentina. E-mail: cristianrabanal@conicet.gov.ar

\section{RESUMEN}

Este artículo investiga la existencia de un ciclo común para los países integrantes de la Asociación Latinoamericana de Integración (ALADI), en el período 1945-2015. Para ello, se considera un modelo factorial, a partir de los componentes cíclicos obtenidos a partir de los filtros de Hodrick-Prescott (HP) y de Baxter-King (BK). Los resultados muestran la existencia de un ciclo común sin Cuba cuando el filtro HP es utilizado, y sin Bolivia, Cuba y Panamá con el procedimiento de BK, aunque una alta correlación entre ambos es encontrada, al tiempo que exhibe una alta conformidad con las principales crisis que han afectado a la región. Por último, el análisis del componente común sugiere que las expansiones han sido más prolongadas que las recesiones, dando cuenta de la asimetría del mismo.

Palabras clave: Sincronización Cíclica, Modelo Factorial, Integración Regional, Filtros.

\section{Cyclical Synchronization in ALADI's Countries}

\begin{abstract}
This paper finds out about a common cycle among ALADI's countries, in the period 1945-2015. In order to do it, a factorial model is taken in count. Also, the Hodrick-Prescott and the Baxter-King filters are considered to obtain each national cyclical component. The results show the existence of a common cycle, without Cuba in the first case, and without Bolivia, Cuba and Panama, when national cycles came from BK filter. There is a high correlation between both common factors. Furthermore, the common cycles reflect the historical crisis in an accurate way. Finally, the analysis suggests that common cycle expansions have been longer than recession, reflecting its asymmetry.

Keywords: Cyclical Synchronization, Factorial Model, Regional Integration, Filters.
\end{abstract}

Clasificación JEL: E32, F15, F41

\footnotetext{
* El autor agradece la participación de la Universidad Nacional de Villa Mercedes, Escuela de Gestión de Empresas y Economía, (c/ Urquiza 72, D5730 Villa Mercedes, Argentina) en la financiación de la investigación para la realización de este estudio. Asimismo, el autor agradece los comentarios de dos evaluadores anónimos.
}

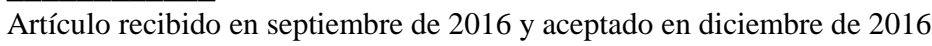

Artículo disponible en versión electrónica en la página www.revista-eea.net, ref. ə-35202 


\section{INTRODUCCIÓN}

El estudio de la sincronización cíclica entre los países pertenecientes a una región es un tema de gran interés entre los economistas por diversas razones. En primer lugar, porque el desarrollo de algunas políticas la presupone. Tal es el caso, por ejemplo, de la adopción de una moneda única. En este sentido, en el modelo de las áreas monetarias óptimas (AMO) desarrollado por Mundell (1961), se espera que las ganancias de la eficiencia monetaria, derivada de unos costos transaccionales menores (producto de la presencia de una sola moneda), sean mayores en la medida que la integración económica también lo sea. Es decir, un mayor número de transacciones garantiza, en dicho caso, beneficios crecientes. Asimismo, se espera que a medida que los procesos de integración avancen, la sincronía cíclica de los países involucrados aumente (Díaz Roldan, 2016).

No obstante, existe una potencial pérdida de eficiencia por parte de cada uno de los países que renuncian a su moneda. La misma, surge a raíz de la imposibilidad de estabilizar sus economías a partir de la política monetaria (Lama y Rabanal, 2014). Sin embargo, esta pérdida de eficiencia será tanto menor en la medida que aumente la integración con el resto de países que comparte la moneda común, pero para que esto suceda será necesario tener ciclos sincronizados.

Otra fuente de gran atención ha sido la cuestión referida a los canales de transmisión de los ciclos económicos internacionales. En particular, existe un fuerte debate sobre la cuestión de los cambios en los patrones de co-movimiento del ciclo económico internacional (Kose et. al, 2008). Por un lado, se encuentran quienes consideran que la globalización ha incrementado la interdependencia de los países, facilitando la convergencia de sus ciclos. Por otra parte, están aquellos que sostienen la teoría del desacoplamiento (decoupling), fundamentalmente de las economías emergentes en relación a las industrializadas. Este último punto de vista fue muy defendido en el año 2008, oportunidad en que los países emergentes resistieron la crisis financiera internacional. Dado que habitualmente, y de manera incorrecta, se utiliza el diferencial de crecimiento entre las economías emergentes y las desarrolladas para estudiar el desacoplamiento (Ruíz, 2009), ya que un diferencial positivo podría deberse a la falta de sincronización cíclica, o bien a la mayor aceleración del crecimiento tendencial de los países rezagados, resulta imperioso analizar la sincronización a partir de una medida de ciclo libre de componente tendencial.

La Asociación Latinoamericana de Integración (ALADI), constituida por el Tratado de Montevideo en 1980, tiene como objetivo final lograr un mercado común a partir de mecanismos de preferencia arancelaria a productos de los países miembros frente a productos de terceros países. La decisión de muchos estados del mundo de sumarse a procesos de integración regional o a mega 
acuerdos comerciales, radica no sólo en la intención de beneficiarse mutuamente de las ganancias teóricas provenientes del comercio y la movilidad de capitales, sino también en el aumento de la cooperación entre países miembros y en la posibilidad de formar un mercado más amplio (Basnet y Sharma, 2013).

Dado que la ALADI es el mayor grupo latinoamericano de integración, es importante analizar la dinámica de los co-movimientos de sus países miembros, ya que resulta una condición fundamental para afrontar un proceso de integración. A pesar de ello, la bibliografía referida a este bloque de países ha sido escasa en relación a otros.

El artículo se propone estudiar la sincronización cíclica de los países pertenecientes a dicha asociación, durante el período 1965-2015. El objetivo específico es identificar, a partir de un modelo factorial, el ciclo común que permita dar cuenta de los principales períodos expansivos y recesivos de los países de la región, esperando encontrar una alta conformidad de ciclo común con los hechos históricos. Desde esta perspectiva, las variables sujetas a análisis son los componentes cíclicos, que serán obtenidos mediante dos procedimientos de filtrado diferentes (Hodrick-Prescott y Baxter-King), con el objetivo de lograr resultados robustos.

Los datos utilizados son los de PBI per cápita, para el período 1965-2015 y provienen del Banco Mundial (World Bank Data), y de estimaciones propias para el año 2015. Las series están expresadas en dólares constantes de 2005, lo que posibilita la comparación.

El trabajo consta de cuatro secciones además de esta. En la segunda, se abordan los principales antecedentes en relación al tema. La tercera sección desarrolla la metodología a utilizar, mientras que la cuarta muestra los principales resultados. La última sección estudia las características del ciclo común estimado, y por último se presentan las consideraciones finales.

\section{NATURALEZA DE LA SINCRONIZACIÓN CÍCLICA. ANTECEDENTES}

Son diversos los motivos que pueden dar lugar a la sincronización cíclica para un conjunto de economías. En primer lugar, las economías en cuestión pueden tener un alto grado de intercambio comercial. En este sentido, la evidencia muestra que la relación directa entre comercio e integración, señalada por la teoría económica, implica ciclos altamente correlacionados entre países que son socios comerciales (Baxter y Kouparitzas, 2004; Arora y Vamvakidis, 2005). También Akin (2006), sugiere que los procesos de integración crean comercio, lo que además de aumentar los vínculos comerciales, incrementa el grado de sincronización cíclica entre las economías participantes de los acuerdos comerciales. Sin embargo, existen algunas excepciones, de casos paradigmáticos, como por ejemplo el de México y Estados Unidos que presentan ambigüedad en 
algunas variables (Mejía Reyes, et. al., 2006), a pesar del fuerte intercambio entre estos dos estados.

No obstante, Böwer y Guillemineau (2006) advierten que a pesar de que la intensificación de comercio entre dos países es, a menudo, señalado como el principal driver para el incremento de la sincronización cíclica, a nivel teórico subsiste aún una controversia. Por un lado, se puede afirmar que efectivamente el aumento en la intensidad del comercio produce unos ciclos más sincronizados, lo que se da necesariamente en virtud de las externalidades producidas por las innovaciones tecnológicas. En contrapartida, otra corriente liderada por Krugman (1992), señala que en realidad el aumento del comercio puede dar lugar a una mayor especialización por parte de los países que intervienen. En consecuencia, el resultado sobre la sincronización, dependerá, de acuerdo a esta corriente, de la característica del comercio, es decir si es intraindustrial o interindustrial, siendo este último el predominante en casos de especialización. Esta distinción ha dado lugar al reconocimiento de la especialización en la producción como una fuente de sincronización, siendo la relación entre ambas inversa.

Otro canal comúnmente señalado como factor generador de sincronización ha sido la integración financiera. No obstante, este tema también se encuentra sujeto a debate. En ese sentido, la evidencia empírica reciente sugiere que la integración financiera puede, en realidad, desincronizar los productos brutos nacionales en relación al ciclo mundial (Monnet y Puy, 2016). Esta idea es contraria a la de Kose et. al. (2008), quienes sostienen la existencia de una relación positiva entre integración financiera y sincronización, desarrollada básicamente vía externalidades.

Imbs (2004), en un intento por integrar las tres fuentes tradicionalmente consideradas en relación a la sincronización cíclica, estima un modelo multiecuacional, encontrando evidencia de que los patrones de especialización tienen un considerable efecto negativo sobre la sincronización. Al mismo tiempo, el autor confirma, a partir de una variedad de medidas de integración financiera, el vínculo positivo con la sincronización. Por último, la integración comercial promueve el incremento de co-movimientos, pero el influjo en relación a la especialización sería neutro.

Las investigaciones para América Latina, han sido referidas en general a un país específico o para cortos períodos de tiempo (Aiolfi, et. al., 2011). En este sentido, la disponibilidad de trabajos que analizan la sincronización cíclica de países de Latinoamérica a partir de modelos factoriales es escasa o se refiere a un grupo acotado de países. Aiolfi, et. al. (2011) construyen un índice de ciclo económico para las economías de Argentina, Brasil, Chile y México.

Algunos autores como Basnet y Sharma (2013), han estudiado el proceso de integración en América Latina, a partir del análisis de las siete mayores economía de la región (Argentina, Brasil, Chile, Colombia, México, Perú y 
Venezuela). Encuentran evidencia de que dichos países presentan no sólo una tendencia común, si no que comparten ciclos comunes. Además del PBI, los autores estudian el comportamiento del comercio intrarregional, la inversión y el consumo, señalando que el aumento del co-movimiento entre las mayores economías, resulta un aliciente para que las menores puedan sumarse a los procesos de integración.

\section{METODOLOGÍA}

La metodología a utilizar, proporcionada por el análisis factorial, constituye una herramienta útil para la detección de un ciclo común entre un grupo de países. Dicho procedimiento ha sido popularizado para el estudio de los ciclos comunes por Stock y Watson (1998, 2002).

Para comenzar, se parte de considerar una tabla de datos como la siguiente:

Tabla 1

Matriz de datos

\begin{tabular}{|ccccccccccccc|}
\hline \multirow{2}{*}{ Año } & Obs. & \multicolumn{10}{c|}{ Variables } \\
\cline { 3 - 11 } & $\mathrm{X}_{\mathbf{1}}$ & $\mathrm{X}_{\mathbf{2}}$ & $\mathrm{X}_{\mathbf{3}}$ & $\mathrm{X}_{\mathbf{4}}$ & $\mathrm{X}_{\mathbf{5}}$ & $\ldots$ & $\mathrm{X}_{\mathbf{9}}$ & $\mathrm{X}_{\mathbf{1 0}}$ & $\mathrm{X}_{\mathbf{1 1}}$ & $\mathrm{X}_{\mathbf{1 2}}$ & $\mathrm{X}_{\mathbf{1 3}}$ \\
\hline 1950 & 1 & $\mathrm{X}_{1,1}$ & $\mathrm{X}_{1,2}$ & $\mathrm{X}_{1,3}$ & $\mathrm{X}_{1,4}$ & $\mathrm{X}_{1,5}$ & $\ldots$ & $\mathrm{X}_{1,9}$ & $\mathrm{X}_{1,10}$ & $\mathrm{X}_{1,11}$ & $\mathrm{X}_{1,12}$ & $\mathrm{X}_{1,13}$ \\
1951 & 2 & $\mathrm{X}_{2,1}$ & $\mathrm{X}_{2,2}$ & $\mathrm{X}_{2,3}$ & $\mathrm{X}_{2,4}$ & $\mathrm{X}_{2,5}$ & $\ldots$ & $\mathrm{X}_{2,9}$ & $\mathrm{X}_{2,10}$ & $\mathrm{X}_{2,11}$ & $\mathrm{X}_{2,12}$ & $\mathrm{X}_{2,13}$ \\
$\ldots$ & 3 & $\mathrm{X}_{3,1}$ & $\mathrm{X}_{3,2}$ & $\mathrm{X}_{3,3}$ & $\mathrm{X}_{3,4}$ & $\mathrm{X}_{3,5}$ & $\ldots$ & $\mathrm{X}_{3,9}$ & $\mathrm{X}_{3,10}$ & $\mathrm{X}_{3,11}$ & $\mathrm{X}_{3,12}$ & $\mathrm{X}_{3,13}$ \\
& $\ldots$ & $\ldots$ & $\ldots$ & $\ldots$ & $\ldots$ & $\ldots$ & $\ldots$ & $\ldots$ & $\ldots$ & $\ldots$ & $\ldots$ & $\ldots$ \\
2015 & $t$ & $\mathrm{X}_{\mathrm{t}, 1}$ & $\mathrm{X}_{\mathrm{t}, 2}$ & $\mathrm{X}_{\mathrm{t}, 3}$ & $\mathrm{X}_{\mathrm{t}, 4}$ & $\mathrm{X}_{\mathrm{t}, 5}$ & $\ldots$ & $\mathrm{X}_{\mathrm{t}, 9}$ & $\mathrm{X}_{\mathrm{t}, 10}$ & $\mathrm{X}_{\mathrm{t}, 11}$ & $\mathrm{X}_{\mathrm{t}, 12}$ & $\mathrm{X}_{\mathrm{t}, 13}$ \\
\hline
\end{tabular}

$X_{1}$ es el ciclo de Argentina, $X_{2}$ el ciclo de Bolivia, $X_{3}$ el ciclo de Brasil, $X_{4}$ el ciclo de Chile, $X_{5}$ el ciclo de Colombia, $X_{6}$ el ciclo de Cuba, $x_{7}$ el ciclo de Ecuador, $X_{8}$ el ciclo de México, $X_{9}$ el ciclo de Panamá, $X_{10}$ el ciclo de Paraguay, $X_{11}$ el ciclo de Perú, $X_{12}$ el ciclo de Uruguay y $X_{13}$ el ciclo de Venezuela.

Fuente: Elaboración propia.

La Tabla 1 puede resumirse en una matriz $\boldsymbol{X}_{\text {txp }}$, siendo $t$ las filas los individuos o períodos temporales considerados, y $p$ las columnas las variables consideradas. De esta forma, se tiene $t$ períodos de tiempo y $p$ variables (trece en este caso). En consecuencia, el ciclo de cada país será explicado por un conjunto de factores, que reducen la información de todas las variables.

En el contexto de este trabajo, se utilizarán componentes cíclicos procedentes de aplicar el filtro de Hodrick-Prescott (1997) -HP en adelante- y el de BaxterKing (1999) -BK en adelante-, sobre la serie PBI per cápita a dólares constantes del año 2005, en orden a obtener estimaciones robustas. Para el caso del filtro HP se utilizará un valor de $\lambda=100$, ya que dicho valor aproxima bien a un filtro ideal. En el anexo se presentan los ciclos de cada uno de los países analizados, bajo ambos procedimientos. Dichos filtros han sido utilizados por una parte importante del análisis empírico sobre ciclos económicos (Rabanal y Baronio, 2010; Mejía y Campos, 2011; Salá Ríos, et. al., 2011). 
Sintéticamente, el problema de minimización planteado en el filtro HodrickPrescott es el siguiente:

$$
\operatorname{Min}_{\left\{\tau_{t}\right\}_{t=1}^{T}}=\sum_{t=1}^{T}\left(y_{t}-\tau_{t}\right)^{2}
$$

sujeto a

$$
\sum_{t=2}^{T-1}\left[\left(\tau_{t+1}-\tau_{t}\right)-\left(\tau_{t}-\tau_{t-1}\right)\right]^{2} \leq \mu
$$

Es evidente que si $\mu=0$ se está en presencia de Mínimos Cuadrados ${ }^{1}$.

Por su parte, el filtro de Baxter-King cuenta fundamentalmente con dos etapas: en la primera una medición del ciclo es realizada, mientras que posteriormente se aísla el componente cíclico y el tendencial mediante la aplicación de medias móviles. En este caso, el problema radica en la elección de una cantidad adecuada de rezagos. La inclusión de rezagos adicionales acerca el filtro a uno ideal, pero lo hace a costa de una pérdida creciente de datos. Por la forma en la que el filtro es construido, se perderán datos al comienzo y al final de la serie, con una cantidad total igual a dos veces la cantidad de rezagos de la media móvil adoptada, lo que constituye una de sus mayores debilidades. ${ }^{2}$.

De esta forma, el análisis factorial propuesto procura estimar el siguiente modelo:

$$
\begin{aligned}
& x_{1}=\beta_{11} f_{1}+\beta_{12} f_{2}+\ldots .+\beta_{1 k} f_{k}+\varepsilon_{1} \\
& x_{2}=\beta_{21} f_{1}+\beta_{22} f_{2}+\ldots .+\beta_{2 k} f_{k}+\varepsilon_{2} \\
& x_{3}=\beta_{31} f_{1}+\beta_{32} f_{2}+\ldots .+\beta_{3 k} f_{k}+\varepsilon_{3} \\
& \ldots \\
& x_{t}=\beta_{t 1} f_{1}+\beta_{t 2} f_{2}+\ldots .+\beta_{t k} f_{k}+\varepsilon_{t}
\end{aligned}
$$

Donde $f_{1}, f_{2}, \ldots, f_{k}(k<t)$ son los factores comunes, $\beta_{i j}(i=1,2, \ldots, t \mathrm{y}$ $j=1,2, \ldots, k)$ las cargas factoriales, y $\varepsilon_{i}$ los factores específicos. En términos matriciales, la expresión (3) equivale a:

$$
\boldsymbol{X}=\boldsymbol{B F}+\boldsymbol{E}
$$

\footnotetext{
${ }^{1}$ El supuesto clave del filtro, consiste en permitir un desvío estándar del cinco por ciento para el componente cíclico a lo largo de toda la serie y de un medio para las segundas diferencias, lo que posibilita computar los valores de $\lambda$ sugeridos por Hodrick y Prescott como:

$$
\lambda^{1 / 2}=\frac{\sigma_{i}}{\sigma_{j}}
$$

donde $\sigma_{\mathrm{i}}$ es el desvío estándar del componente cíclico $\sigma_{\mathrm{j}}$ el desvío estándar de las segundas diferencias. Un análisis más detallado del filtro puede encontrarse en Hodrick y Prescott (1997).

2 El lector interesado puede encontrar una breve referencia del procedimiento en Rabanal y Baronio (2010) o una completa explicación en Baxter y King (1999).
} 
Siendo $\boldsymbol{X}$ la matriz de datos, $\boldsymbol{B}$ representa la matriz de cargas factoriales, $\boldsymbol{F}$ la matriz de factores y $\boldsymbol{E}$ el vector de residuos.

Dada la naturaleza de los componentes cíclicos, $x_{1}, x_{2}, \ldots, x_{t}$, los mismos tendrán media nula. Dichos componentes, pueden ser expresados como una combinación lineal de $k$ factores comunes a los ciclos de todos los países analizados. Tal como señala De Lucas (2008), esos factores deben cumplir con una serie de supuestos:

$$
\begin{gathered}
E(\boldsymbol{F})=0 \\
E\left(\boldsymbol{F} \boldsymbol{F}^{\prime}\right)=\boldsymbol{I} \\
E(\boldsymbol{E})=0 \\
E\left(E E^{\prime}\right)=\boldsymbol{\Omega} \\
E(\boldsymbol{F E})=0
\end{gathered}
$$

siendo $\boldsymbol{I}$ la matriz de varianzas y covarianzas de los factores comunes. Dado que es una matriz identidad, se asume que la covarianza entre los diferentes factores comunes es nula. $\boldsymbol{\Omega}$ es una matriz diagonal de varianzas y covarianzas de los residuos con ceros fuera de la diagonal principal, por lo que los factores idiosincráticos de los diferentes países no podrán estar correlacionados. Asimismo, se supone que tanto los factores como los residuos tienen media nula, mientras que la ecuación (9) implica que el factor cíclico común no puede estar correlacionado con el factor cíclico idiosincrático de cada país.

La suma de las cargas factoriales asociadas a cada variable elevadas al cuadrado se denominan comunalidades. Estas expresan la proporción de la variación de cíclica de un país en cuestión explicada por los factores. La diferencia entre la variación total y la comunalidad se denomina especificidad, y refiere al movimiento cíclico autónomo de cada país.

Por lo tanto, una de las cuestiones más relevantes en el análisis factorial, radica en la elección del número de factores a incluir. Dado que la regla general exige parsimonia e interpretabilidad del modelo, el número final de factores considerados debe ser el mínimo posible. No obstante, esta determinación ha dado lugar a una extensa bibliografía al respecto.

En este trabajo, se adopta el análisis de Factores Principales. Dicho procedimiento proporciona, en la práctica, soluciones similares al Análisis de Componentes Principales, al tiempo que los parámetros son en ambos casos igualmente generalizables (Velicer y Jackson, 1990).

El número de factores será determinado de acuerdo con el criterio de KaiserGuttman. Esta técnica, sugerida originariamente por Guttman (1954), y adaptada posteriormente por Kaiser (1961), establece que deben considerarse aquellos factores que tengan asociados valores propios mayores a la unidad. No obstante, dado que el procedimiento basado exclusivamente en criterio de Kaiser-Guttman 
puede dar lugar a la inclusión de un número excesivo de factores, también se considera la elección del número de factores en relación al criterio de parsimonia, que implica elegir aquellos factores situados antes del punto de inflexión en el gráfico de sedimentación (Scree Plot). Es decir, se consideran aquellos factores con valores propios asociados mayores a la unidad, pero hasta el punto de inflexión del Scree Plot. Por otra parte, siguiendo el análisis propuesto por De Lucas (2008), para el caso del ciclo económico internacional, aquellos países que muestren alguna carga factorial inferior a 0.4 serán eliminados, y se reestimará el modelo.

La validación del modelo propuesto exigirá que la adecuación muestral de los datos sea mayor a 0.5, lo justifica emplear el procedimiento de análisis factorial. En particular, se utilizará el índice de Kaiser-Meyer-Olkin (KMO), que puede expresarse como sigue:

$$
K M O=\frac{\sum \sum_{i \neq j} r_{j i}^{2}}{\sum \sum_{i \neq j} r_{j i}^{2}+\sum \sum_{i \neq j} a_{j i}^{2}}
$$

siendo $r_{i j}$ los coeficientes de correlación obtenidos entre las variables originales y $a_{i j}$ los coeficientes de correlación parcial entre las variables originales. Cuanto más próximo a uno sea el valor del índice, mayor será la adecuación del conjunto de datos al modelo factorial propuesto.

Adicionalmente, se evaluará la bondad del ajuste de los modelos propuestos mediante el Índice de Ajuste Normado de Bentler-Bonet (IANBB). La ventaja de este índice sobre las tradicionales pruebas de chi-cuadrado radica en que el mismo permite controlar el efecto que el número de individuos tiene sobre la significatividad de la prueba chi-cuadrado. El IANBB asume valores entre cero y uno, y aunque no existe una regla fija, se consideran aceptables los valores por encima de 0.85 .

Finalmente, cada uno de los componentes cíclicos comunes, obtenidos a partir de cada procedimiento de filtrado, será analizado en sus características. Para ello, se utilizará el algoritmo propuesto por Bry-Boschan (1971). Dichas rutinas serán ejecutadas en el toolbox Grocer de Scilab.

\section{RESULTADOS}

En la Tabla 2 se resumen los modelos estimados para el caso de los componentes extraídos a partir del filtro HP.

Esta tabla muestra, en primer lugar, que se pueden considerar prescindibles los factores 3 y 4, ya que además de contar con cargas factoriales bajas, reúnen una varianza desdeñable. Adicionalmente, resultan contrarios a los principios de 
parsimonia e interpretabilidad. Asimismo, puede notarse en la Figura 8 (ver anexo) que existen dos factores antes del punto de inflexión en el Scree Plot.

Tabla 2

Modelo factorial inicial. Ciclos HP

\begin{tabular}{|c|c|c|c|c|c|c|}
\hline \multirow{2}{*}{ País } & \multicolumn{4}{|c|}{ Cargas factoriales } & \multirow{2}{*}{ Comunalidad } & \multirow{2}{*}{ Especificidad } \\
\hline & F1 & F2 & F3 & F4 & & \\
\hline Argentina & 0,68762 & 0,258363 & 0,371835 & 0,209048 & 0,721535 & 0,278465 \\
\hline Bolivia & 0,549377 & $-0,346787$ & $-0,379375$ & 0,166063 & 0,593579 & 0,406421 \\
\hline Brasil & 0,529335 & $-0,425003$ & 0,343957 & $-0,205449$ & 0,621339 & 0,378661 \\
\hline Chile & 0,563206 & 0,492464 & $-0,116812$ & $-0,398498$ & 0,732167 & 0,267833 \\
\hline Colombia & 0,803596 & $-0,150296$ & 0,024198 & $-0,144439$ & 0,689803 & 0,310197 \\
\hline Cuba & $-0,378957$ & $-0,110464$ & 0,187619 & $-0,104371$ & 0,201905 & 0,798095 \\
\hline Ecuador & 0,522255 & $-0,608316$ & $-0,179459$ & 0,294853 & 0,761943 & 0,238057 \\
\hline México & 0,404495 & 0,438425 & $-0,369867$ & 0,206897 & 0,535441 & 0,464559 \\
\hline Panamá & 0,32481 & 0,571529 & 0,225358 & 0,370026 & 0,619853 & 0,380147 \\
\hline Paraguay & 0,774808 & 0,071211 & $-0,364188$ & $-0,239921$ & 0,795594 & 0,204406 \\
\hline Perú & 0,426002 & $-0,049494$ & 0,494578 & $-0,10574$ & 0,439716 & 0,560284 \\
\hline Uruguay & 0,851772 & 0,049129 & $-0,004651$ & $-0,110633$ & 0,740191 & 0,259809 \\
\hline Venezuela & 0,665172 & $-0,123024$ & 0,200992 & 0,213533 & 0,543582 & 0,456418 \\
\hline
\end{tabular}

Adecuación muestral de Kaiser: 0.6854. IANBB: 0.9823. Varianza acumulada por factor: F1: 0.581875, F2: 0.776823, F3: 0.912803 y F4: 1.

Fuente: Elaboración propia con datos de World Bank Data.

Por otro lado, el IANBB es de 0.98 , con lo cual presenta una bondad de ajuste satisfactoria y los primeros dos factores relevantes para el análisis, reúnen una varianza acumulada cercana al 78\%. No obstante, los resultados sugieren que Cuba ha desarrollado un ciclo cuyo comportamiento ha resultado prácticamente idiosincrático en su totalidad e inconexo al de los restantes países de la ALADI, lo cual resulta congruente con la inmovilidad de capitales y las restricciones comerciales que rigieron en el país durante el período de estudio de este trabajo.

Asimismo, en función de las cargas factoriales para los dos primeros factores, se vislumbra que Uruguay, Colombia, Paraguay, Argentina y Venezuela son los países con mayores cargas, respectivamente, en el primer factor. Por otra parte, muchos países que aún teniendo cargas "aceptables" (mayor a 0.4) en el primer factor, mantienen en el segundo cargas significativas. Tal es el caso de Brasil, Chile, Ecuador, México y Panamá, lo que indica que este grupo de países presentan mayores variaciones cíclicas idiosincráticas, o que se ajustan en menor medida al ciclo común.

Por otra parte, y para el caso de los ciclos generados a partir del filtro BK, ilustrados en la Tabla 3 a continuación, los resultados indican que ni Bolivia, Cuba, y Panamá se ajustan al ciclo común representado por el primer factor. Nótese también, que el segundo factor es prescindible, pues sólo un país (Argentina) muestra una carga factorial significativa. 
Tabla 3

Modelo factorial inicial. Ciclos BK

\begin{tabular}{|ccccccc|}
\hline \multirow{2}{*}{ País } & F1 & Cargas factoriales & & Comunalidad & Especificidad \\
& F2 & F3 & F4 & & \\
\hline Argentina & 0,536485 & $-0,474639$ & 0,055107 & 0,091555 & 0,524517 & 0,475483 \\
Bolivia & 0,26386 & 0,389386 & 0,232402 & $-0,059158$ & 0,278754 & 0,721246 \\
Brasil & 0,608951 & 0,07483 & $-0,295422$ & $-0,261744$ & 0,532205 & 0,467795 \\
Chile & 0,573008 & 0,141361 & $-0,219691$ & 0,33382 & 0,508021 & 0,491979 \\
Colombia & 0,635855 & 0,260047 & 0,083582 & $-0,031562$ & 0,479918 & 0,520082 \\
Cuba & $-0,262218$ & 0,356449 & 0,300884 & 0,073435 & 0,291738 & 0,708262 \\
Ecuador & 0,436859 & 0,210271 & 0,347172 & $-0,285665$ & 0,437193 & 0,562807 \\
México & 0,515216 & 0,056307 & 0,096908 & 0,339127 & 0,393017 & 0,606983 \\
Panamá & 0,351099 & $-0,264213$ & 0,207666 & 0,160188 & 0,261864 & 0,738136 \\
Paraguay & 0,648288 & 0,380564 & $-0,276673$ & 0,10017 & 0,651689 & 0,348311 \\
Perú & 0,618072 & $-0,197783$ & $-0,122432$ & $-0,365967$ & 0,570053 & 0,429947 \\
Uruguay & 0,778543 & $-0,162158$ & $-0,018483$ & 0,035225 & 0,634007 & 0,365993 \\
Venezuela & 0,447684 & $-0,162811$ & 0,526195 & 0,003539 & 0,503822 & 0,496178 \\
\hline
\end{tabular}

Adecuación muestral de Kaiser: 0.6896. IANBB: 0.9657. Varianza acumulada por factor: F1: 0.612856, F2: 0.769629, F3: 0.906754 y F4: 1.

Fuente: Elaboración propia con datos de World Bank Data.

En consecuencia, ambas estimaciones iniciales precisan ser reestimadas en función de que los factores 3 y 4 no son relevantes para el primer caso, mientras que para el segundo caso no resultan valiosos los factores 2,3 y 4 .

Para el caso de los ciclos obtenidos desde el filtro HP, se elimina Cuba, ya que su ciclo no consigue una carga factorial de 0.4 en ninguno de los primeros dos factores, y los factores 3 y 4 en línea con el criterio de parsimonia, dando lugar al modelo final presentado en la Tabla 4.

Tabla 4

Modelo factorial final. Ciclos HP

\begin{tabular}{|ccccc|}
\hline \multirow{2}{*}{ País } & \multicolumn{2}{c}{ Cargas factoriales } & Comunalidad & Especificidad \\
\hline Argentina & 0,681158 & 0,269853 & 0,536797 & 0,463203 \\
Bolivia & 0,539308 & $-0,354754$ & 0,416703 & 0,583297 \\
Brasil & 0,539783 & $-0,411622$ & 0,460798 & 0,539202 \\
Chile & 0,551748 & 0,48946 & 0,543997 & 0,456003 \\
Colombia & 0,80543 & $-0,14279$ & 0,669107 & 0,330893 \\
Ecuador & 0,52047 & $-0,606022$ & 0,638152 & 0,361848 \\
México & 0,39848 & 0,436592 & 0,349399 & 0,650601 \\
Panamá & 0,312829 & 0,571474 & 0,424445 & 0,575555 \\
Paraguay & 0,776173 & 0,076512 & 0,608298 & 0,391702 \\
Perú & 0,43828 & $-0,024206$ & 0,192676 & 0,807324 \\
Uruguay & 0,853805 & 0,062524 & 0,732892 & 0,267108 \\
Venezuela & 0,671689 & $-0,096543$ & 0,460487 & 0,539513 \\
\hline
\end{tabular}

Adecuación muestral de Kaiser: 0.707631. IANBB: 0.9169.

Fuente: Elaboración propia con datos de World Bank Data. 
Este modelo final, compuesto por dos factores, permite explicar porcentajes que van desde el cuarenta hasta el setenta por ciento, aproximadamente, del movimiento cíclico de los países a partir del ciclo común, excepto para el caso de México y Perú, cuyos países presentan elevadas especificidades. Asimismo, existe una alta adecuación muestral que justifica la reducción de la información a estos dos factores. La misma resulta ser de 0.71. La bondad del ajuste del modelo final es de 0.91 .

En la Tabla 5 se muestran las estimaciones finales para el componente cíclico obtenido a través del filtrado pasa banda, y atendiendo las modificaciones necesarias de acuerdo al análisis de la Tabla 3.

Tabla 5

Modelo factorial final. Ciclos BK

\begin{tabular}{|cccc|}
\hline País & $\begin{array}{c}\text { Cargas factoriales } \\
\text { F1 }\end{array}$ & Comunalidad & Especificidad \\
\hline Argentina & 0,521579 & 0,272045 & 0,727955 \\
Brasil & 0,59714 & 0,356576 & 0,643424 \\
Chile & 0,579592 & 0,335926 & 0,664074 \\
Colombia & 0,639997 & 0,409597 & 0,590403 \\
Ecuador & 0,416532 & 0,173499 & 0,826501 \\
México & 0,518684 & 0,269033 & 0,730967 \\
Paraguay & 0,646105 & 0,417451 & 0,582549 \\
Perú & 0,622765 & 0,387836 & 0,612164 \\
Uruguay & 0,775861 & 0,60196 & 0,39804 \\
Venezuela & 0,452787 & 0,205016 & 0,794984 \\
\hline
\end{tabular}

Adecuación muestral de Kaiser: 0.713135. IANBB: 0.8841.

Fuente: Elaboración propia con datos de World Bank Data.

Este procedimiento reduce la información a un solo factor, con una adecuación muestral alta de 0.71 y con un IANBB de 0.88. Lógicamente, esto repercute en comunalidades menores. Para todos los países (salvo Uruguay), es la especificidad la que explica en mayor medida la evolución cíclica de cada uno de los países. La exclusión de Bolivia, en este segundo procedimiento, es consistente con los resultados obtenidos por Díaz Roldan (2016), quien analizando el carácter y la temporalidad de las perturbaciones que han afectado a un grupo de países (de la Comunidad Andina, MERCOSUR y sus asociados), a partir de la metodología sugerida por Cohen y Wyplosz (1989), encuentra un grado de temporalidad muy alto para cualquier tipo de perturbación.

Es preciso destacar la pérdida de información al comienzo y al final de cada una de las series, dada la estructura del filtro BK.

En ambos modelos finales, es Uruguay la economía de referencia, vale decir la que presenta un menor comportamiento idiosincrático. También coinciden 
ambos modelos en asignarles altas cargas a Colombia y Paraguay, lo cual se aprecia claramente en los mapas de cuantiles mostrados en la Figura 1 y 2.

Finalmente, la rotación de factores realizada mediante el método Varimax Ortogonal conduce a cargas factoriales rotadas similares a las presentadas en la Tabla 4, a excepción de Argentina que presenta mayor carga en el segundo factor (F2). Para el modelo final obtenido a partir de ciclos BK no es posible realizar la rotación, en virtud de que el modelo final retiene un único factor.

Figura 1

Cuantiles para F1. Ciclos HP
Figura 2

Cuantiles para F1. Ciclos BK

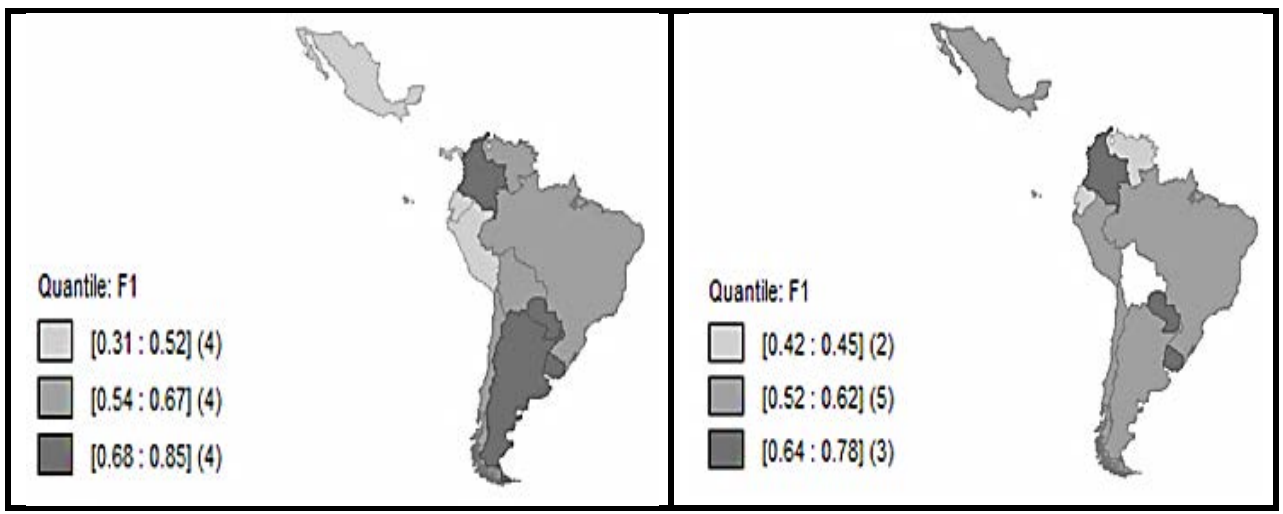

Fuente: Elaboración propia.

\section{ANÁLISIS DEL CICLO COMÚN}

En la sección anterior se obtuvieron dos modelos alternativos, para representar el ciclo común de los países integrantes de la ALADI. Si bien el generado a partir de los componentes cíclicos de BK no tiene en cuenta la contribución de Bolivia, Cuba, ni Panamá, mientras que el análisis del ciclo común obtenido desde el filtro HP deja de lado sólo a Cuba, se puede observar, en primer lugar que sus performances, a lo largo del período analizado, son similares en términos generales. De hecho, el coeficiente de correlación de ambos es de 0.83. La Figura 3 permite visualizar que la diferencia, entre uno y otro, está dada básicamente en la intensidad y no en la dirección de los movimientos. También es necesario tener en cuenta que el filtro BK pierde datos al comienzo y al final de la serie por la forma en la que se encuentra construido.

Existe además una conformidad alta y razonable de los mismos en relación a los hechos históricos que afectaron a Latinoamérica. Esto es, en ambos casos, el ciclo común refleja satisfactoriamente las principales etapas de expansión y recesión de los países estudiados a lo largo del período 1965-2015, lo cual no 
resulta sorprendente, ya que muchos de los shocks que afectaron a la región fueron de carácter global. Algunos ejemplos de ello son la suba del precio del petróleo en 1973, la crisis de la deuda de comienzos de los ochenta, la denominada burbuja de las puntocom y la crisis financiera internacional del 2008.

Figura 3

Evolución del componente cíclico común. BK versus HP

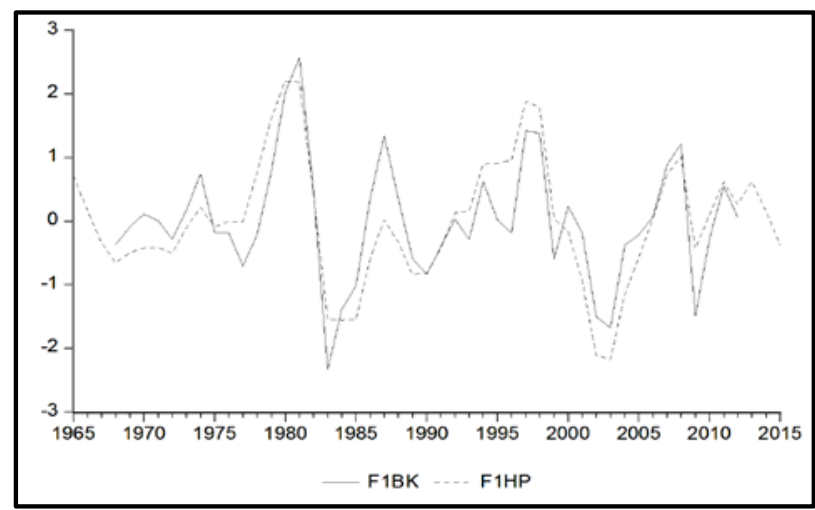

Fuente: Elaboración propia.

\subsection{Fechado cíclico y características del ciclo común}

El análisis del ciclo común se lleva adelante mediante la utilización del algoritmo propuesto por Bry-Boschan (1971), de gran aceptación entre los investigadores en virtud de su capacidad para reproducir la cronología cíclica de Estados Unidos difundida por la National Bureau of Economic Research (NBER) con gran exactitud.

Figura 4

Puntos de giro para F1. Ciclos HP. Fechado Bry-Boschan

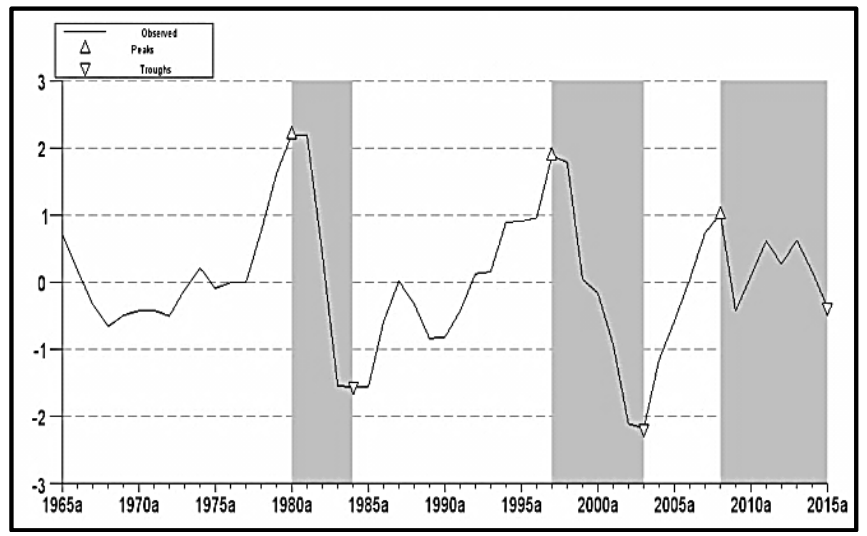

Fuente: Elaboración propia. Software. Scilab / Grocer. 
Para el primer caso, que considera los componentes cíclicos de cada país obtenidos por el filtro HP, se determinaron los puntos de giro correspondiente al primer factor. La Figura 4 ilustra los mismos.

Como se puede apreciar, el algoritmo de fechado capta satisfactoriamente las principales crisis durante el período de estudio. Sin embargo, el procedimiento no alcanza a identificar la crisis ocurrida en la década del setenta, con la suba del precio del petróleo. Adicionalmente, la Tabla 6 presentada a continuación, resume la cronología cíclica del ciclo común obtenido desde componentes cíclicos HP, y la Tabla 7 presenta las principales características.

Tabla 6

Puntos de giro. Ciclo HP de F1. Procedimiento de Bry-Boschan

\begin{tabular}{|ccccc|}
\hline \multicolumn{2}{c}{ Puntos de Giro } & \multicolumn{3}{c|}{ Duraciones } \\
Picos & Valles & Expansión & Contracción & Ciclo \\
\hline & 1968 & $\ldots$ & $\ldots$ & $\ldots$ \\
1980 & 1984 & 12 & 4 & 16 \\
1997 & 2003 & 13 & 6 & 19 \\
2008 & & 5 & $\ldots$ & $\ldots$ \\
\hline
\end{tabular}

Fuente: Elaboración propia.

Los comienzos de las crisis son datadas en 1980, 1997 y 2008, mientras que los comienzos de las expansiones para el ciclo común son en los años 1968, 1984 y 2003.

La Tabla 7 muestra claramente la asimetría del ciclo común, dado que presenta una duración promedio de pico a valle de cinco años, mientras de valle a pico resulta ser el doble, de diez años.

Tabla 7

Duración y amplitud de ciclo HP de F1

\begin{tabular}{|cc|}
\hline Duración Promedio & Valor (Años) \\
\hline De pico a pico & 14 \\
De valle a valle & 17,5 \\
De pico a valle & 5 \\
De valle a pico & 10 \\
\hline Amplitud Promedio & Valor (eje Y Factor1) \\
\hline De pico a valle & $-3,91$ \\
De valle a pico & 3,16 \\
\hline
\end{tabular}

Fuente: Elaboración propia.

Para finalizar, la Figura 5 ilustra los puntos de giro del componente común resultante del modelo resumido en la Tabla 5, y en la Tabla 8 se tabulan dichos valores. 
Figura 5

Puntos de giro para F1. Ciclos BK. Fechado Bry-Boschan

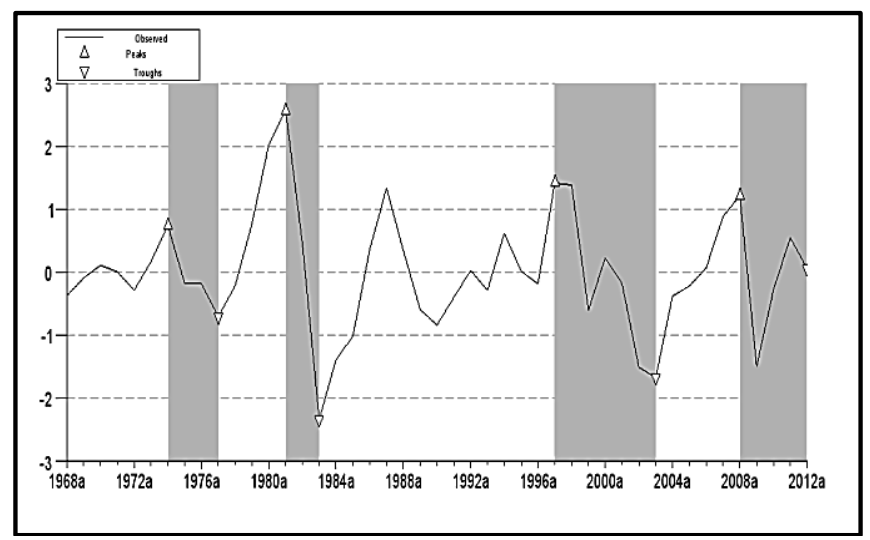

Fuente: Elaboración propia. Software. Scilab / Grocer.

Tabla 8

Puntos de giro. Ciclo BK de F1. Procedimiento de Bry-Boschan

\begin{tabular}{|ccccc|}
\hline \multicolumn{2}{c}{ Puntos de Giro } & \multicolumn{3}{c|}{ Duraciones } \\
Picos & Valles & Expansión & Contracción & Ciclo \\
\hline 1974 & 1977 & $\ldots$ & 3 & $\ldots$ \\
1981 & 1983 & 4 & 2 & 6 \\
1997 & 2003 & 14 & 6 & 20 \\
2008 & & 5 & $\ldots$ & $\ldots$ \\
\hline
\end{tabular}

Fuente: Elaboración propia.

A diferencia del ciclo común presentado en el primer caso (HP), en éste se identifica la crisis en la década del setenta, cuyo comienzo es el año 1974 y cuyo final es 1977. También existe una ligera diferencia en la crisis del ochenta, con fecha de comienzo en 1981 y fecha de final en 1983.

Tabla 9

Duración y amplitud de ciclo BK de F1

\begin{tabular}{|cc|}
\hline Duración Promedio & Valor (Años) \\
\hline De pico a pico & 11,33 \\
De valle a valle & 13 \\
De pico a valle & 3,67 \\
De valle a pico & 7,66 \\
\hline Amplitud Promedio & Valor (eje Y Factor $\mathbf{1}$ ) \\
\hline De pico a valle & $-3,15$ \\
De valle a pico & 3,3 \\
\hline
\end{tabular}

Fuente: Elaboración propia. 
Finalmente, Tabla 9 permite dar cuenta de que, al igual que en el anterior caso, el componente común tiene una marcada asimetría, aunque más acotada en esta oportunidad.

\section{CONCLUSIONES}

En este trabajo se presenta un análisis de sincronización cíclica entre los países miembros de la Asociación Latinoamericana de Integración (ALADI), que constituye el mayor bloque de integración de la región.

El principal aporte de esta investigación radica en la obtención de un ciclo común, a partir de la propuesta metodológica sugerida por Stock y Watson $(1998,2002)$. Con el propósito de obtener estimaciones robustas, se extrajeron los componentes cíclicos de cada país con dos procedimientos diferentes: el filtro HP y el de BK. En ambos casos, el filtrado se llevó a cabo con valores propicios de los parámetros, para que aproximaran a un filtro ideal.

Para el primer caso (filtro HP), el modelo factorial propuesto favoreció la inclusión de dos factores, los que en conjunto explicaban cerca del $80 \%$ de la varianza. Sólo un país, Cuba, debió ser descartado para la reestimación del modelo en virtud de sus bajas cargas factoriales.

Por otra parte, el segundo modelo basado en los componentes cíclicos de BK arrojó sólo un factor relevante, concentrando el $60 \%$ de la varianza. En esta oportunidad, la reestimación exigió retirar del cálculo a Bolivia, Cuba y Panamá por cargas factoriales poco significativas.

Ambos presentaron una elevada adecuación muestral de los datos, lo que justifica el empleo de la técnica adoptada.

Posteriormente, se analizó la evolución del ciclo común en cada caso, encontrando gran similitud entre ambos procedimientos y presentando un correlato con las principales crisis de carácter global que azotaron a la región.

El algoritmo de Bry-Boschan (1974) fue utilizado para la obtención de la cronología cíclica del ciclo común. Esto permitió establecer las fechas de comienzo y fin de las recesiones y expansiones, las que en general fueron coincidentes con ambas formas de filtrar los datos. La diferencia más notable en este sentido fue la detección de la crisis de los setenta con el ciclo común BK, que no fue debidamente captada en el caso de HP.

Finalmente, en relación a la duración del ciclo común, ambas formas de filtrar arriban a un ciclo que tiene como característica principal la asimetría, fenómeno que refleja que las duraciones son más prolongadas que las recesiones.

El presente estudio podría ser ampliado en futuras líneas de investigación a partir del empleo de técnicas procedentes de un modelo factorial dinámico múltiple. 


\section{REFERENCIAS BIBLIOGRÁFICAS}

AIOLFI, M., CATÃO, L. A., y TIMMERMANN, A. (2011). "Common factors in Latin America's business Cycles". Journal of Development Economics, 95 (2), pp. 212-228.

AKIN, C. (2006). Multiple Determinants of Business Cycle Synchronization. Montreal: Annual Meeting of the Canadian Economic Association, Montreal.

ARORA, V. y VAMVAKIDIS, A. (2005). "How much do Trading Partners Matter for Economic Growth?". Journal of Economic Integration, 52, pp. 24-40.

BASNET, H. C. y SHARMA, S. C. (2013). "Economic Integration in Latin America". Journal of Economic Integration. 28(4). Pp. 551-579.

BAXTER, M. y KING R. G. (1999). "Measuring Business Cycles: Approximate Band-Pass Filters For Economic Time Series". The Review of Economics and Statistics, MIT Press. Vol. 81(4), pp. 575-593.

BAXTER, M. y KOUPARITZAS, M. (2004). "Determinants of Business Cycle Comovement: A Robust Analysis". National Bureau of Economic Research, Working Paper 10725.

BÖWER, U. y GUILLEMINEAU, C. (2006). "Determinants of Business Cycle Synchronization Across Euro Area Countries". Working Paper Series, 587, European Central Bank.

BRY, G. y BOSCHAN C. (1971). "Cyclical Analysis of Time Series: Selected procedures and computer programs". NBER, New York, NY.

COHEN, D. y C. WYPLOSZ. (1989). "The European Monetary Union: An Agnostic evaluation. En R. Bryant et. al. (eds.): Macroeconomic policies in an interdependent world. International Monetary Fund, Washington, DC, 311- 337.

DELAJARA, M. (2013). "Comovimiento y concordancia cíclica del empleo en los Estados Mexicanos". Economía mexicana. Nueva época, 22(2), pp. 297-340.

DE LUCAS, S. (2008). "Ciclo y Convergencia: una perspectiva mundial, 1950-2006". Tesis Doctoral.

DÍAZ ROLDÁN, C. (2016). "Las perturbaciones macroeconómicas en Latinoamérica: implicaciones en los procesos de integración económica y monetaria". Revista de Economía Mundial.42, pp. 19-42.

GUTTMAN, L. (1954). "Some Necessary Conditions for Common-Factor Analysis". Psychometrika, Vol. 19(2), pp. 149-161.

HARDING, D. y PAGAN, A. (1999). "Dissecting the cycle". Working Paper, Melbourne Institute, University of Melbourne, (13/99).

HODRICK, R. J. y PRESCOTT, E. C. (1997). "Postwar U.S Business Cycles: An Empirical Investigation". Journal of Money, Credit and Banking. 29, pp. 1-16.

IMBS, J. (2004). "Trade, finance, specialization, and synchronization". Review of Economics and Statistics, 86 (3), pp. 723-734.

KAISER,H . F. (1961). "A note on Guttman'sl Ower Bound for the Number of Common Factors". British Journial of Psychology, 14, pp. 1-2.

KOSE, M. A., OTROK, C., \& PRASAD, E. S. (2008). "Global Business Cycles: Convergence or Decoupling?". National Bureau of Economic Research (No. w14292).

KRUGMAN, Paul (1992). "Lessons of Massachusetts for EMU". En Torres, F. y Francesco Giavazzi (eds.): Adjustment and Growth in the European Monetary Union, Cambridge: Cambridge University Press, pp. 193-229. 
LAMA, R. y RABANAL, P. (2014). "Deciding to Enter a Monetary Union: The Role of Trade and Financial Linkages". European Economic Review, 72, pp. 138-165.

MEJÍA REYES, P.; GUTIÉRREZ ALVA, E. E.; FARÍAS, S. y CLAUDIA, A. (2006). "La sincronización de los ciclos económicos de México y Estados Unidos Investigación Económica". Investigación Económica, 65 (258), pp. 15-45.

MEJÍA, P. y J. CAMPOS-CHÁVEZ (2011). "Are the Mexican States and the United States bussines cycles synchoronized?evidence from the manufacturing production". Economía Mexicana, 20 (1), pp. 79-112.

MONNET, E. y PUY, D. (2016). "Has Globalization Really Increased Business Cycle Synchronization?". IFM Working Paper, 16 (54), pp. 1-55.

MUNDELL, R. (1961). "A Theory of Optimum Currency Areas". American Economic Review, Vol. 51, pp. 657-665.

RABANAL, C. y BARONIO, A. M. (2010). "Alternativas para la modelización de tendencias y ciclos en la economía argentina, 1880-2009". Estudios de Economía Aplicada, 28 (3), pp. 651-670.

RODRIK D. (2002). "Feasible Globalizations”. NBER Working Paper, No. 9129.

RUIZ, À. (2009). "¿El retorno del decoupling? Mito y realidad en el desacoplamiento de las economías emergentes". Documentos de Economía "La Caixa", 16, pp. 1-30.

SALÁ RÍOS, M., FARRÉ PERDIGUER, M. y TORRES SOLÉ, T. (2011). "El ciclo económico de Cataluña. Un análisis de la simetría respecto a España y la UEM". Estudios de Economía Aplicada, 29 (3), pp. 1-24.

STOCK, J. H., \& WATSON, M. W. (1998). "Diffusion indexes". National Bureau of Economic Research. (No. w6702).

STOCK, J. y WATSON, M. (2002). "Forecasting Using Principal Components From a Large Number of Predictors". Journal of American Statistical Association. 97 (460), pp. 1167-1179.

VELICER, W. F. y JACKSON, D. N. (1990). "Component Analysis versus Common Factor Analysis: Some issues in Selecting an Appropriate Procedure". Multivariate Behavioral Research, 25 (1), pp. 1-28. 


\section{ANEXO}

Figura 6

Componentes cíclicos HP. Log. del PBI per cápita en dólares de 2005
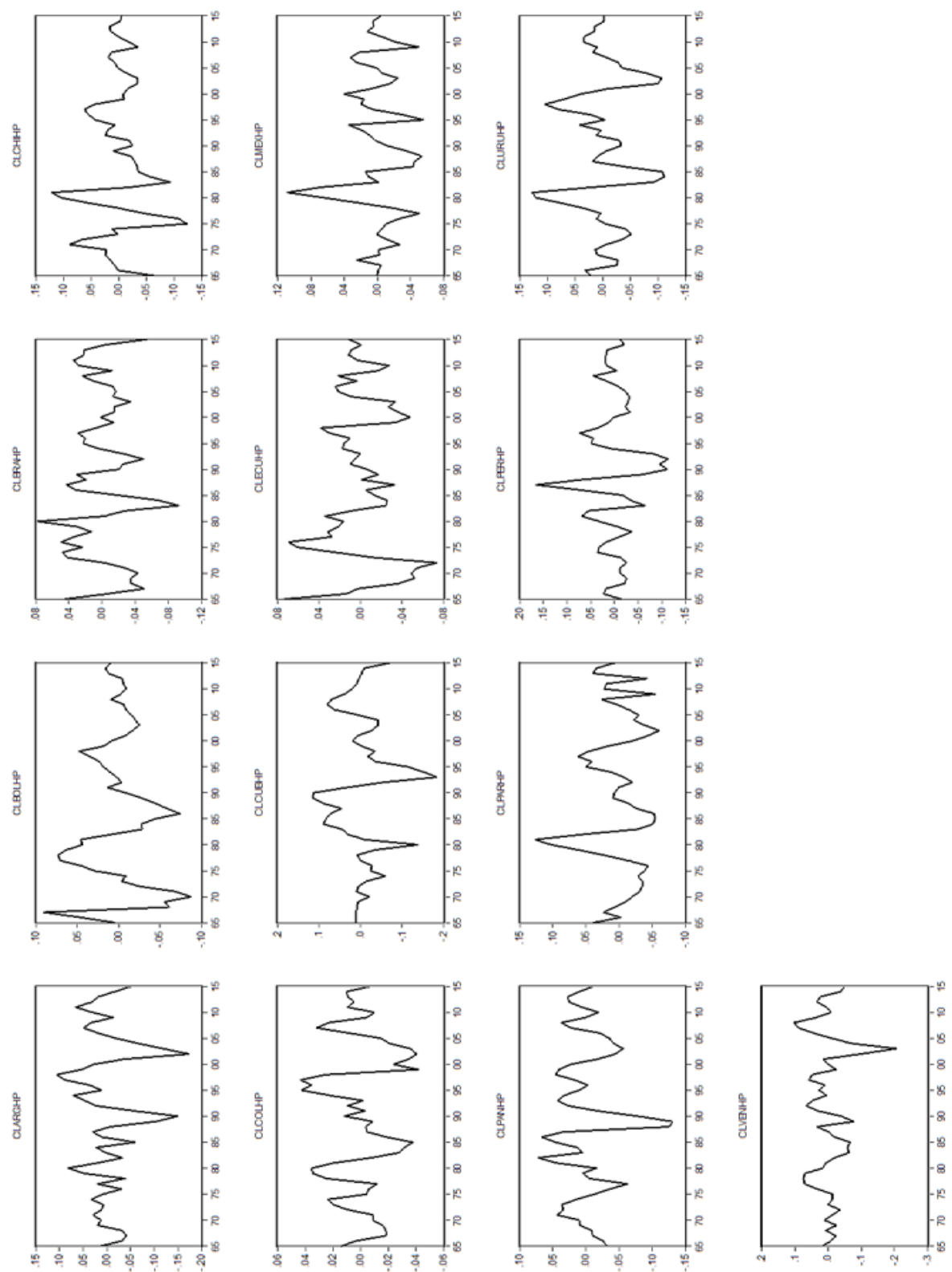

Fuente: Elaboración propia. 
Figura 7

Componentes cíclicos BK. Log. del PBI per cápita en dólares de 2005
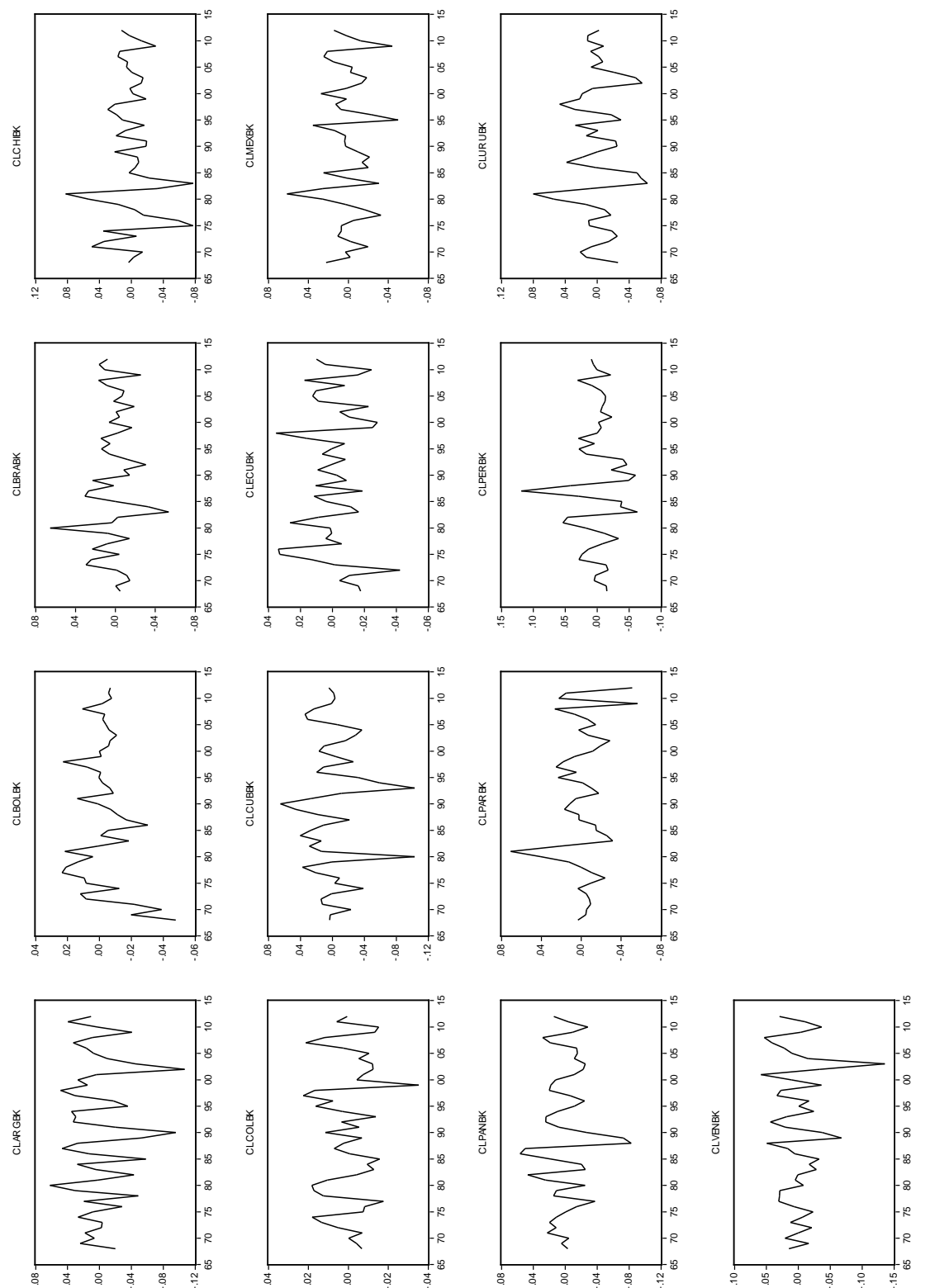

Fuente: Elaboración propia. 


\section{Figura 8}

Scree Plot del modelo factorial inicial. Ciclos HP. Valores propios (ordenada) vs. número de factores

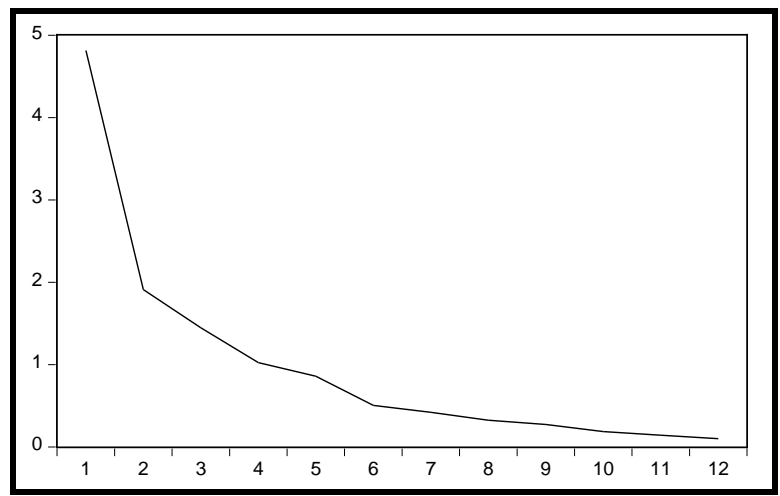

Fuente: Elaboración propia.

Figura 9

Scree Plot del modelo factorial inicial. Ciclos BK. Valores propios (ordenada) vs. número de factores

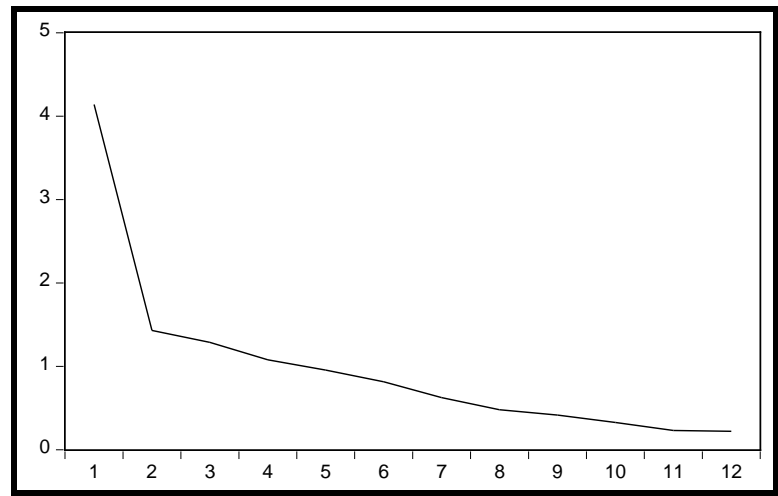

Fuente: Elaboración propia. 
